\title{
Subspace Identification of Hammerstein Systems with Nonparametric Input Backlash and Switch Nonlinearities
}

\author{
Mathieu Pouliquen, Fouad Giri, Olivier Gehan, \\ Eric Pigeon, Miloud Frikel, Boubekeur Targui \\ Control Group, GREYC CNRS UMR 6072 \\ ENSICAEN, 06 Bd du Marechal Juin \\ 14050 Caen Cedex, France \\ mathieu.pouliquendunicaen.fr
}

\begin{abstract}
In this paper, a subspace identification algorithm for a class of Hammerstein systems is developed. We consider dynamical systems subject to input backlash or switch nonlinearities. The idea is to use a specific input signal allowing the estimation of the nonlinear part and the estimation of a state space model for the linear part. The identification algorithm is a subspace type algorithm. A simulation example is given to illustrate the performances of the present method.
\end{abstract}

\section{INTRODUCTION}

The Hammerstein systems are the nonlinear systems consisting of a nonlinearity block followed by a linear dynamic system. The identification of such systems has received much interest for the last decades as evidenced by the many following references: [22], [6], [1], [7], [34], [12], [32], [35], [2], [11], [25], [31], [8], [13], [20], [16] and the four sessions devoted to block oriented nonlinear identification at SYSID 2012. This interest is justify by the fact that these systems take into account nonlinearities commonly encountered in practice, generated by the technological limitations necessary for the proper functioning of the system (saturation, the limit stops, etc.). In this paper we consider the identification in presence of some particular input nonlinearities: backlash and switch nonlinearities.

Few contributions deal with the identification of system with input backlash or switch nonlinearity. The approaches developed in [1], [4], [12] and [5] are devoted to nonlinearities bordered by straight lines. The method introduced in [25] is adapted to nonlinearities with more general borders however the proposed method is a two experiments method. Moreover it uses Least Squares algorithm coupled to an overparameterization, which leads to the need for a high number of data. An other method is also depicted in [13]. Nevertheless this method is only adapted to backlash nonlinearity and it is a two stages method using two independent experiments.

In this paper, we propose an approach which alleviates some of the issues present in the previous methods. First, our method is devoted to backlash and switch nonlinearities with general borders. Second, our method is a single stage method which allows to estimate both the linear subsystem and the nonlinearity. Let us notice that the borders of the nonlinearities are nonparametric, they will be piecewise parameterized as in [27].
The proposed identification method is based on two key idea: (1) A specific input sequence is design in order to allow a linear parameterization of the identification problem and in order to satisfy the persistent excitation condition. (2) Subspace identification methods are used for the estimation. The use of such methods is mainly motivated by a set of interesting properties: the simplicity, the intrinsic numerical robustness and their straightforward application to MIMO systems ([26], [29], [30], [10], [18], [23], [24], [9]). Some subspace methods adapted to Hammerstein systems have been introduced ([15], [14], [3], [33], [28], [17], [21]) but, to the best of our knowledge, these methods aren't adapted to the case of backlash or switch nonlinearity.

The outline of this paper is as follows: in section II the model structure is presented and the specific input signal is designed. In section III the subspace identification algorithm is applied. Some simulation results are given in section IV. Finally, section V concludes the paper.

\section{PROBLEM STATEMENT AND PARAMETRIZATION}

In this section we first state the identification problem. Then we described the design of the excitation signal. The association of that signal with a parametrization of the nonlinearity yields a linear identification problem.

\section{A. Identification problem statement}

Consider the identification of the Hammerstein system shown in Fig. 1 where $u(t)$ and $y(t)$ are respectively the input and the output of the plant which is expressed as:

$$
y(t)=G(q) H[u(t)]+v(t)
$$

$G(q)$ is the proper transfer function of the deterministic linear part in the Hammerstein system and $v(t)$ is an additive noise on the output. $v(t)$ is wide sense stationary zero mean process uncorrelated with $u(t)$. The nonlinear part in the Hammerstein system is described by $H[$.$] . This function can be a$ switch or a backlash element. Its borders are characterized by the descendant and ascendent continuous borders $H_{d}[$. and $H_{a}[$.$] . These two nonlinearities are illustrated on Fig. 2$ and Fig. 3. 


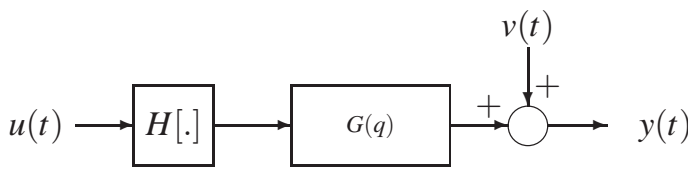

Fig. 1. Considered Hammerstein system

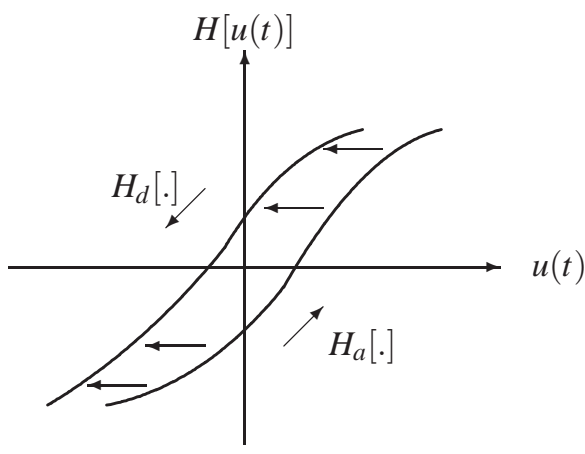

Fig. 2. Backlash nonlinearity

The identification problem treated in this paper is stated as: based on the input-output measurements, find the minimal order $n$ of the system, a realization of state space matrices $(A, B, C, D)$ for $G(q)$ and a nonparametric description of the descendant and ascendent borders $H_{d}[$.$] and H_{a}[$.$] . The$ identification of the nonlinearity will be performed on a working interval: $u(t) \in\left[u_{\min } ; u_{\max }\right]$.

So as to be able to solve the identification problem we make some assumptions throughout the paper:

A.1 In the case of the switch nonlinearity we have $H_{a}\left[u_{\min }\right]=H_{d}\left[u_{\min }\right]$

A.2 The static gain of $G(q)$ is unitary: $G(1)=1$;

Remark 1: The assumption $[A .2]$ is necessary so as to satisfy the identifiability condition. Without this assumption we are not able to distinguish the solution $G(q) H[u(t)]$ from the solution $(a G(q))\left(\frac{1}{a} H[u(t)]\right)$ with $a \neq 0$.

\section{B. Design of the excitation signal and parametrization of the identification problem}

In this subsection first we describe the suggested excitation signal, then we use it with a piecewise parameterization of the nonlinearity.

The design of the input signal $u(t)$ is the corner stone of the identification method proposed in this paper. A major difficulty is to select an excitation satisfying the persistent excitation condition and allowing a characterization of the

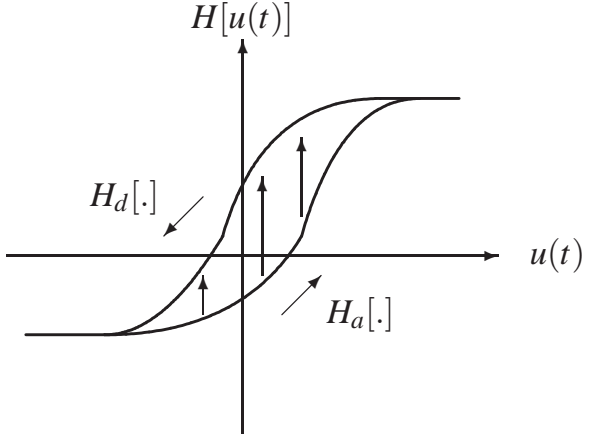

Fig. 3. Switch nonlinearity

Input design procedure

- The initial value is $u(0)=u_{\min }$

- For $t \geq 1$, follow the following cycle:

step $1 u(t)$ equal to a random value in $] u_{\min } ; u_{\max }$ [ (we take the ascending path) then $t=t+1$;

step $2 u(t)=u_{\max }$ (we continue the ascending path until $\left.u_{\max }\right)$ then $t=t+1$

step $3 u(t)$ equal to a random value in $] u_{\min } ; u_{\max }$ [ (we take the descending path) then $t=t+1$;

step $4 u(t)=u_{\min }$ (we continue the descending path until $u_{\text {min }}$ ) then $t=t+1$ and go back to step 1 .

TABLE I

ascendant and descendant paths. Such an excitation is illustrated on Fig. 4 and table I presents its design procedure.

This excitation signal has two key properties. Firstly it produces persistent excitation on the input of the system thanks to the random value one sample on two. Secondly $u(t)$ browses continuously in their entirety the ascending and descending paths. Such an excitation ensures cycles described on Figs. 2 and 3 to be driven always in the same manner between $u_{\min }$ and $u_{\max }$. If $u_{\min }$ and $u_{\max }$ are constant (we assume they are), these paths are unique for the backlash nonlinearity and the switch nonlinearity.

Remark 2: In order to characterize homogeneously functions $H_{a}[$.$] and H_{d}[$.$] on \left[u_{\min } ; u_{\max }\right]$, we recommend choosing a random value uniformly distributed over this interval.

Let us denote respectively $P_{a}[$.$] and P_{d}[$.$] the ascending$ and descending path i.e. the extension of the borders $H_{a}[$. and $H_{d}[$.$] in the following manner:$

- if $u(t)$ is increasing then $H[u(t)]=P_{a}[u(t)]$;

- if $u(t)$ is decreasing then $H[u(t)]=P_{d}[u(t)]$.

At any time $t$ it is possible to know which path uses $H[u(t)]$. To this end, let define a direction signal $\delta(t)$ which 


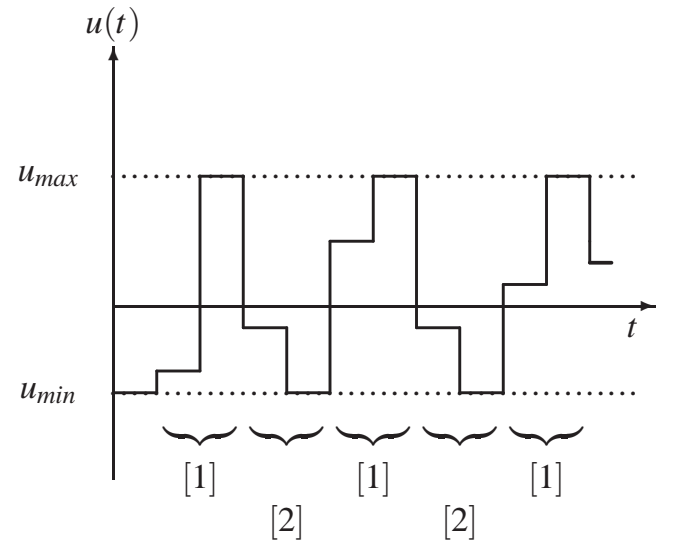

Fig. 4. The excitation signal is divided into two phases: the first [1] in which we take the ascending path, the second [2] for which we take the descending path.

indicates if $u(t)$ is increasing or decreasing:

$$
\delta(t)=\left\{\begin{array}{l}
1 \text { if } u(t)>u(t-1) \\
\delta(t-1) \text { if } u(t)=u(t-1) \\
0 \text { if } u(t)<u(t-1)
\end{array}\right.
$$

From this definition, $H[u(t)]$ can be formulated as follows

$$
H[u(t)]=\delta(t) P_{a}[u(t)]+(1-\delta(t)) P_{d}[u(t)]
$$

So as to characterize the functions $P_{a}[u(t)]$ and $P_{d}[u(t)]$, we approximate them by continuous piecewise linear functions as in [27]. These approximations consist in a sum of functions $\left\{P_{i}^{a}().\right\}$ for the ascending path and a sum of functions $\left\{P_{i}^{d}().\right\}$ for the descending path in the following manner:

$$
\left\{\begin{array}{l}
P_{a}[u(t)]=\mu_{0}^{a}+\mu_{1}^{a} P_{1}^{a}(u(t))+\mu_{2}^{a} P_{2}^{a}(u(t))+\cdots+\mu_{n_{P}}^{a} P_{n_{P}}^{a}(u(t)) \\
P_{d}[u(t)]=\mu_{0}^{d}+\mu_{1}^{d} P_{1}^{d}(u(t))+\mu_{2}^{d} P_{2}^{d}(u(t))+\cdots+\mu_{n_{P}}^{d} P_{n_{P}}^{d}(u(t))
\end{array}\right.
$$

with $\mu_{0}^{a}=P_{a}\left(u_{\min }\right), \mu_{0}^{d}=P_{d}\left(u_{\min }\right)$ and

$$
P_{i}^{a}(u(t))= \begin{cases}0 & \text { if } u(t) \leq n_{i-1}^{a} \\ u(t)-n_{i-1}^{a} & \text { if } n_{i-1}^{a} \leq u(t) \leq n_{i}^{a} \\ n_{i}^{a}-n_{i-1}^{a} & \text { if } n_{i}^{a} \leq u(t)\end{cases}
$$

Fig. 5 illustrates such function $P_{i}^{a}(u(t)) \cdot n_{P}+1$ represents the number of nodes between $u_{\min }$ and $u_{\max } . n_{P}+1$ is a user defined index which depends on the application considered as the distributions of the nodes $\left\{n_{i}^{a}\right\}$ and $\left\{n_{i}^{d}\right\}$. Theses nodes can be arbitrarily selected by the user on the interval $\left[u_{\min } ; u_{\max }\right]$.

Remark 3: For ease of notations we have chosen the same number of knots for the ascending path and the descending path, however they could be different.

From (2) and (3) we get

$H[u(t)]=\mu_{0}^{a} \delta(t)+\mu_{0}^{d}(1-\delta(t))+\sum_{i=1}^{n P} \mu_{i}^{a} P_{i}^{a}(u(t)) \delta(t)+\sum_{i=1}^{n P} \mu_{i}^{d} P_{i}^{d}(u(t))(1-\delta(t))$

$$
P_{i}^{a}(u(t))
$$

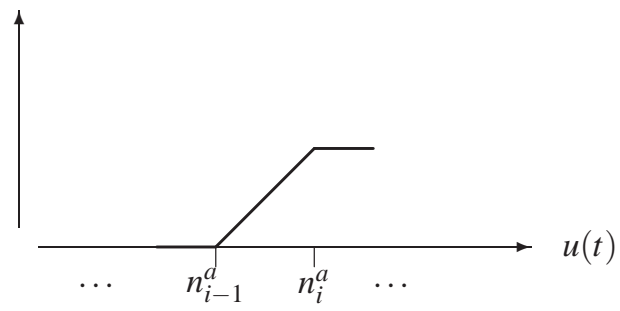

Fig. 5. The function $P_{i}^{a}(u(t))$ for the approximation on the segment $\left[n_{i-1}^{a} ; n_{i}^{a}\right]$

and according assumption $A .1$ whatever the nonlinearity we have $\mu_{0}^{a}=\mu_{0}^{d}$, this gives

$H[u(t)]=\mu_{0}^{a}+\sum_{i=1}^{n_{P}} \mu_{i}^{a} P_{i}^{a}(u(t)) \delta(t)+\sum_{i=1}^{n_{P}} \mu_{i}^{d} P_{i}^{d}(u(t))(1-\delta(t))$

and then

$$
H[u(t)]=\mu z(t)+\mu_{0}^{a}
$$

with

$$
z(t)=\left(\begin{array}{c}
P_{1}^{a}(u(t)) \delta(t) \\
P_{2}^{a}(u(t)) \delta(t) \\
\vdots \\
P_{n_{P}}^{a}(u(t)) \delta(t) \\
P_{1}^{d}(u(t))(1-\delta(t)) \\
P_{2}^{d}(u(t))(1-\delta(t)) \\
\vdots \\
P_{n_{P}}^{d}(u(t))(1-\delta(t))
\end{array}\right)
$$

and

$$
\mu=\left(\begin{array}{llllllll}
\mu_{1}^{a} & \mu_{2}^{a} & \cdots & \mu_{n_{P}}^{a} & \mu_{1}^{d} & \mu_{2}^{d} & \cdots & \mu_{n_{P}}^{d}
\end{array}\right)
$$

From assumptions $A .2$ and making use previous notations in (1) yields

$$
y(t)-\mu_{0}^{a}=G(q) \mu z(t)+v(t)
$$

Let consider here a minimal state space model for $G(q)$, this gives

$$
\left\{\begin{array}{c}
x(t+1)=A x(t)+B \mu z(t) \\
y(t)-\mu_{0}^{a}=C x(t)+D \mu z(t)+v(t)
\end{array}\right.
$$

where $x(t) \in \mathbb{R}^{n}$ is the state vector with $n$ the minimal order, $A \in \mathbb{R}^{n \times n}, B \in \mathbb{R}^{n}, C \in \mathbb{R}^{n}$ and $D \in \mathbb{R}$. $(A, C)$ is assumed to be observable and $(A, B)$ is assumed to be controllable.

From (6) it is possible to define an over-parameterized state space model in the following manner:

$$
\left\{\begin{array}{c}
x(t+1)=A x(t)+\mathbf{B} z(t) \\
y(t)-\mu_{0}^{a}=C x(t)+\mathbf{D} z(t)+v(t)
\end{array}\right.
$$


with $\mathbf{B}=B \mu$ and $\mathbf{D}=D \mu$. This corresponds to a linear state space model with the input $z(t) \in \mathbb{R}^{2 n_{P}}$ and the output $y(t)-$ $\mu_{0}^{a}$. In the next section this over-parameterized model is used in the identification step of the approach.

\section{SUBSPACE IDENTIFICATION ALGORITHM}

As in [33], we use here standard subspace algorithms for the identification of the state space matrix of (7). Input and output are respectively $z(t)$ and $y(t)-\mu_{0}^{a}$, consequently $\mu_{0}^{a}$ is supposed to be known. To this end we recommend the application of an input sequence equal to $u_{\min }$ for a few moments before the application of the excitation sequence described in subsection II-B. The mean of the corresponding output gives $\mu_{0}^{a}$. In the following, let us denote $\bar{y}(t)=y(t)-$ $\mu_{0}^{a}$.

Standard subspace algorithms split the available data into block matrices: output Hankel matrices $\bar{Y}_{p}$ and $\bar{Y}_{f}$ are defined as

$$
\left(\bar{Y}_{p}\right)=\left(\begin{array}{cccc}
\bar{y}(t-p) & \bar{y}(t-p+1) & \ldots & \bar{y}(t-p+j-1) \\
\bar{y}(t-p+1) & \bar{y}(t-p+2) & \ldots & \bar{y}(t-p+j) \\
\ldots & \ldots & \ldots & \ldots \\
\bar{Y} & \ldots & \ldots \\
\bar{y}(t-1) & \bar{y}(t) & \ldots & \bar{y}(t+j-2) \\
\hline \bar{y}(t) & \bar{y}(t+1) & \ldots & \bar{y}(t+j-1) \\
\bar{y}(t+1) & \bar{y}(t+2) & \ldots & \bar{y}(t+j) \\
\ldots & \ldots & \ldots & \ldots \\
\bar{y}(t+f-1) & \bar{y}(t+f) & \ldots & \bar{y}(t+f+j-2)
\end{array}\right)
$$

Similar definitions hold for $V_{f}, Z_{f}$ and $Z_{p}$. Subscripts $f$ and $p$ respectively stand for "future" and "past" and are user-defined indexes. $j$ is the number of columns in the Hankel matrices. In the subspace identification framework it is commonly assumed that there are long time series available i.e. $j \gg 1$.

Using these Hankel matrices, the over-parameterized state space model (7) can be represented by the following basic subspace equations:

$$
\left\{\begin{array}{l}
X_{t}=A^{p} X_{t-p}+\Delta_{p} Z_{p} \\
\bar{Y}_{f}=\Gamma_{f} X_{t}+\Xi_{f} Z_{f}+V_{f}
\end{array}\right.
$$

where $X_{t-p}$ and $X_{t}$ are respectively the past and future state vectors of the plant:

$$
\begin{gathered}
X_{t-p}=\left(\begin{array}{cccc}
x(t-p) & x(t-p+1) & \ldots & x(t-p+j-1)
\end{array}\right) \\
X_{t}=\left(\begin{array}{llll}
x(t) & x(t+1) & \ldots & x(t+j-1)
\end{array}\right) \\
\Gamma_{f}=\left(\begin{array}{c}
C \\
C A \\
\ldots \\
C A^{f-1}
\end{array}\right)
\end{gathered}
$$$$
\text { and } \Delta_{p}=\left(\begin{array}{llll}
A^{p-1} \mathbf{B} & \cdots & A \mathbf{B} & \mathbf{B}
\end{array}\right)
$$

are respectively the extended observability matrix and reversed extended controllability matrix of the model (7).$\Xi_{f}$ is defined by

$$
\Xi_{f}=\left(\begin{array}{cccc}
\mathbf{D} & 0 & \cdots & 0 \\
C \mathbf{B} & \mathbf{D} & 0 & \cdots \\
\cdots & \cdots & \cdots & \cdots \\
C A^{f-2} \mathbf{B} & \cdots & C \mathbf{B} & \mathbf{D}
\end{array}\right)
$$

and it corresponds to a lower triangular Toeplitz matrix containing the first $f$ Markov parameters of (7).

From (8), $\bar{Y}_{f}$ can be rewritten as

$$
\bar{Y}_{f}=L_{Z_{f}} Z_{f}+L_{Z_{p}} Z_{p}+\Gamma_{f} A^{p} X_{t-p}+V_{f}
$$

with

$$
\begin{aligned}
& L_{Z_{p}}=\Gamma_{f} \Delta_{p} \\
& L_{Z_{f}}=\Xi_{f}
\end{aligned}
$$

This becomes

$$
\bar{Y}_{f}=L \Phi+\Gamma_{f} A^{p} X_{t-p}+V_{f}
$$

with

$$
L=\left(\begin{array}{cc}
L_{Z_{p}} & L_{Z_{f}}
\end{array}\right) \quad \Phi=\left(\begin{array}{c}
Z_{p} \\
Z_{f}
\end{array}\right)
$$

Most of classical subspace identification methods are based on the estimation of the extended observability matrix $\Gamma_{f}$ ([26]). This can be done making use the projection of $\bar{Y}_{f} \Phi^{T}\left(\Phi \Phi^{T}\right)^{-1} \Phi$ onto the orthogonal complement of the row space of $Z_{f}$ :

$$
\mathscr{O}_{f}=\bar{Y}_{f} \Phi^{T}\left(\Phi \Phi^{T}\right)^{-1} \Phi \Pi_{Z_{f}}^{\perp}
$$

with $\Pi_{Z_{f}}^{\perp}=I_{j}-Z_{f}^{T}\left(Z_{f} Z_{f}^{T}\right)^{-1} Z_{f}$.

In [26] it is shown that if $f, p>n$, if the input $z(t)$ is uncorrelated with the additive noise $v(t)$ and if $z(t)$ is persistently exciting of order $(f+p) 2 n_{P}$ then the singular values decomposition (SVD) of $\lim _{j \rightarrow \infty} \mathscr{O}_{f}$ is given by

$$
\begin{gathered}
\lim _{j \rightarrow \infty} \mathscr{O}_{f}=\left(\begin{array}{cc}
\mathbb{U}_{1} & \mathbb{U}_{2}
\end{array}\right)\left(\begin{array}{cc}
\mathbb{S}_{1} & 0 \\
0 & 0
\end{array}\right)\left(\begin{array}{l}
\mathbb{V}_{1}^{T} \\
\mathbb{V}_{2}^{T}
\end{array}\right) \\
\lim _{j \rightarrow \infty} \mathscr{O}_{f}=\mathbb{U}_{1} \mathbb{S}_{1} \mathbb{V}_{1}^{T}
\end{gathered}
$$

where $\mathbb{S}_{1} \in \mathbb{R}^{n \times n}$. It follows that the (unknown) order of the system is equal to the number of singular values different from zero and the observability matrix $\Gamma_{f}$ can be taken equal to

$$
\Gamma_{f}=\mathbb{U}_{1} T
$$

where $T$ is a similarity transformation.

Remark 4: Some weighting matrices are often added in (11) in order to include some well known subspace methods such that N4SID, PO-MOESP, PI-MOESP, CVA, IVM, etc.. Here (11) corresponds to the PI-MOESP method ([29], [30]).

Once the order and the extended observability matrix estimated (with (13)), we have to find the state matrices $(A, B, C, D)$ and the parameters $\left\{\mu_{i}^{a}\right\}$ and $\left\{\mu_{i}^{d}\right\}$. The following three steps procedure can be considered: 
1) From (13), the system matrices $A$ and $C$ are obtained as follows 1

$$
\begin{gathered}
C=\Gamma_{f}(1,:) \\
A=\left(\underline{\Gamma}_{f}^{T} \underline{\Gamma}_{f}\right)^{-1} \underline{\Gamma}_{f}^{T} \bar{\Gamma}_{f}
\end{gathered}
$$

with $\underline{\Gamma}_{f}=\Gamma_{f}(1: f-1,:)$ and $\bar{\Gamma}_{f}=\Gamma_{f}(2: f,:)$.

2) $\mathbf{B}$ and $\mathbf{D}$ are determined using the following equation $\bar{Y}_{f} \Phi^{T}\left(\Phi \Phi^{T}\right)^{-1} \Phi=L \Phi+\Gamma_{f} A^{p} X_{t-p} \Phi^{T}\left(\Phi \Phi^{T}\right)^{-1} \Phi$

which gives

$$
\left(\Gamma_{f}\right)^{\perp} Y_{f} \Phi^{T}\left(\Phi \Phi^{T}\right)^{-1} \Phi=\left(\Gamma_{f}\right)^{\perp} \Xi_{f}(\mathbf{B}, \mathbf{D}) Z_{f}
$$

Then the procedure given in [26] is used to obtain $\mathbf{B}$ and $\mathbf{D}$.

3) $B$ and $\mu$ are extracted from $\mathbf{B}$ using a second SVD:

$$
\mathbf{B}=\left(\begin{array}{ll}
u_{1}^{\prime} & \mathbb{U}_{2}^{\prime}
\end{array}\right)\left(\begin{array}{cc}
s_{1}^{\prime} & 0 \\
0 & 0
\end{array}\right)\left(\begin{array}{c}
v_{1}^{\prime T} \\
\mathbb{V}_{2}^{\prime} T
\end{array}\right)
$$

This gives

$$
\begin{gathered}
B=\gamma u_{1}^{\prime} s_{1}^{\prime} \\
\left(\begin{array}{cccc}
\mu_{1} & \mu_{2} & \cdots & \mu_{n_{P}}
\end{array}\right)=\frac{1}{\gamma} v_{1}^{\prime T}
\end{gathered}
$$

$\gamma$ is a parameter adjusted so as to ensure a unitary static gain (assumption A.2).

Remark 5: To improve the numerical robustness, once $A$ and $C$ are known, a method is proposed in [33] in order to estimate the matrices $B$ and $\mu$ separately. This method could be used here.

Remark 6: In this paper only the deterministic part of the plant is identified. It is possible to consider a state space model with noise model which can be rewritten in an innovation form as

$$
\left\{\begin{array}{cl}
x(t+1) & =A x(t)+B H[u(t)]+K e(t) \\
y(t) & =C x(t)+D H[u(t)]+e(t)
\end{array}\right.
$$

The stochastic part is described by the matrix $K \in \mathbb{R}^{n}$ and the covariance $\sigma_{e}^{2}=\mathscr{E}\left\{e(t)^{2}\right\}$. These parameters could easily be estimated using a method proposed in [19].

\section{SIMULATION RESULTS}

To investigate the interest of the method, two numerical example are proposed. On these examples we consider a plant whose linear part is described by the following two order transfert function:

$$
G(q)=q^{-1} \frac{0.1994-0.1804 q^{-1}}{1-1.8858 q^{-1}+0.9048 q^{-2}}
$$

\footnotetext{
${ }^{1}$ MATLAB (c) notations
}

$v(t)$ is such that $v(t)=G_{v}(q) e(t)$ where $e(t)$ is a gaussian white noise and the noise model is given by

$$
G_{v}(q)=\frac{1+0.5 q^{-1}}{1-0.85 q^{-1}}
$$

The identification method is applied on the basis of Monte Carlo simulation of 100 experiments. The variance of $e(t)$ is chosen such that signal to noise ratio is $S N R=10 \mathrm{~dB}$. The number of columns in Hankel matrices is $j=4000$.

\section{A. Case 1: a switch nonlinearity}

In this first case the nonlinear part $H[$.$] is a switch$ nonlinearity characterized by the following functions $H_{a}[$. and $H_{d}[$.$] :$

$$
H_{d}[u(t)]= \begin{cases}-1 & \text { if } u(t) \leq-2 \\ \arctan (u(t)+1)-H_{0} & \text { if }-2<u(t) \leq 2 \\ 1 & \text { if } 2<u(t)\end{cases}
$$

and

$$
H_{a}[u(t)]= \begin{cases}-1 & \text { if } u(t) \leq-2 \\ \arctan (u(t)-1)+H_{0} & \text { if }-2<u(t) \leq 2 \\ 1 & \text { if } 2<u(t)\end{cases}
$$

with $H_{0}=\frac{1}{2}(\arctan (3)-\arctan (1))$. These functions are approximated on the interval $[-3 ; 3]$ and we choose a low number of nodes $n_{P}=7$. These nodes are uniformly distributed on $[-3 ; 3]$ and they are the same for the descendant and ascendent paths.

The estimated poles are shown in Fig. 6, the crosses indicate the true pole locations. This figure shows that the proposed method gives good estimation: no bias and low standard-deviation. Such good estimation quality is confirmed by Fig. 7 which shows the frequency response of the true system and the average of the estimated frequency responses. The estimated average nonlinearity is depicted on Fig. 8. The same conclusion as for Fig. 7 holds.

\section{B. Case 2: a backlash nonlinearity}

In this second case the nonlinear part $H$ [.] is a backlash nonlinearity characterized by the following functions $H_{a}[$. and $H_{d}[$.$] :$

$$
H_{d}[u(t)]=\arctan (u(t)+1)
$$

and

$$
H_{a}[u(t)]=\arctan (u(t)-1)
$$

These functions are again approximated on the interval $[-3 ; 3]$ with the same number of nodes: $n_{P}=7$.

The results are given in Figs. 9, 10 and 11. They confirm the previous conclusion: the proposed method works well. It allows an approximation of the nonlinearity and an unbiased estimation of the linear part. 


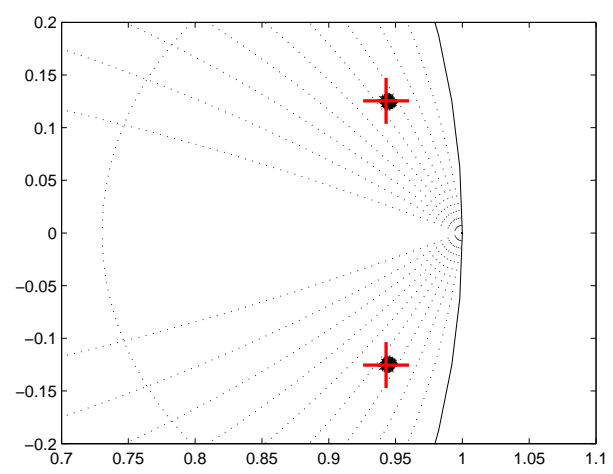

Fig. 6. Switch nonlinearity: the eigenvalues of estimated A matrix: * estimated pole, + system pole.

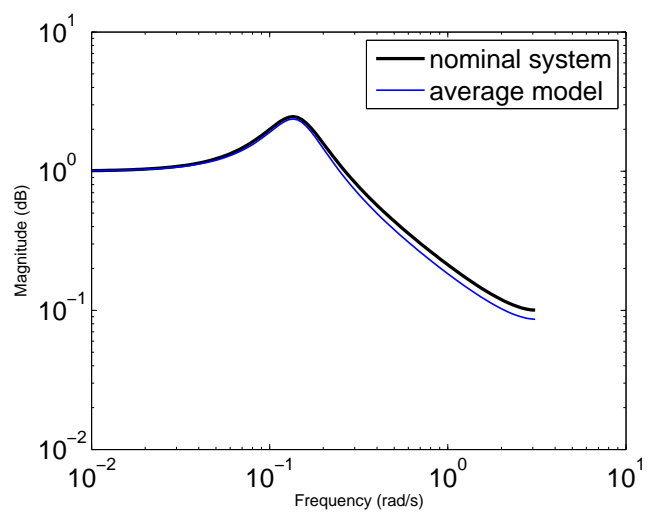

Fig. 7. Switch nonlinearity: the Bode magnitude plot of the system and of the estimated average model

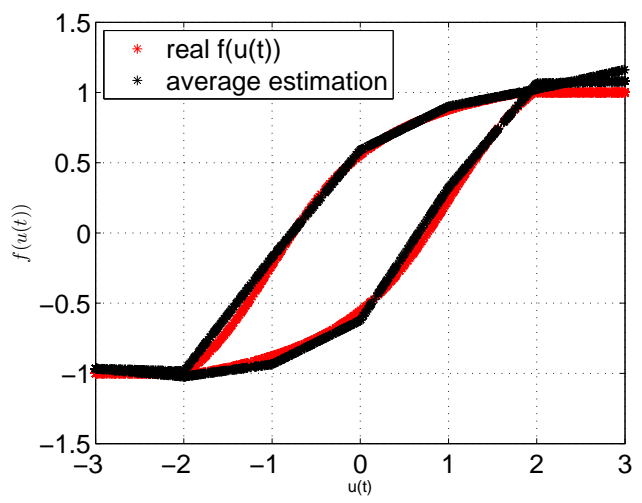

Fig. 8. Switch nonlinearity: H[.] and its average estimation

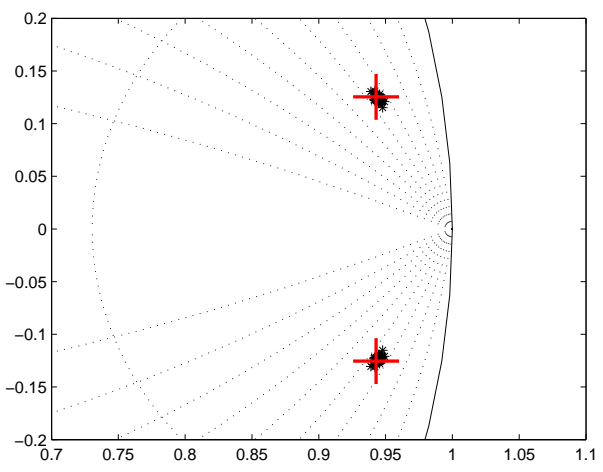

Fig. 9. Backlash nonlinearity: the eigenvalues of estimated A matrix: * estimated pole, + system pole.

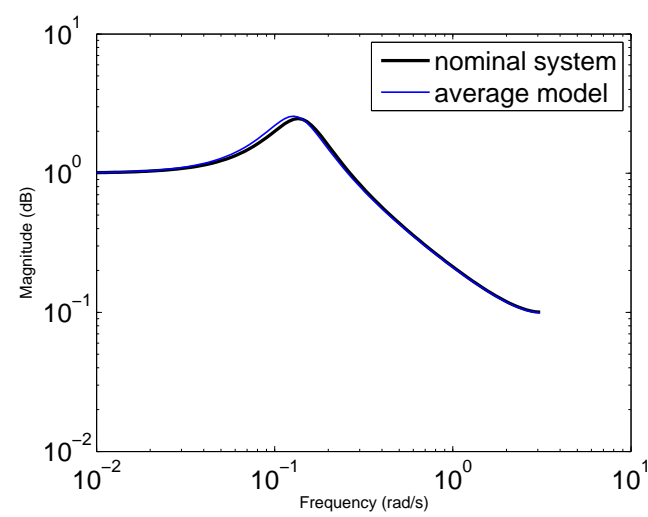

Fig. 10. Backlash nonlinearity: the Bode magnitude plot of the system and of the estimated average model

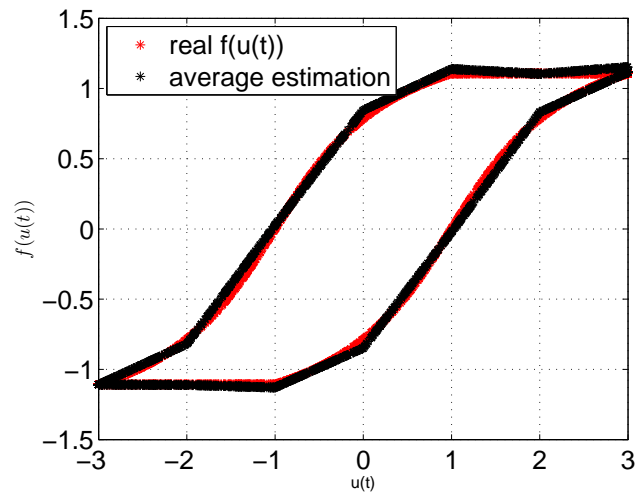

Fig. 11. Backlash nonlinearity: H[.] and its average estimation 


\section{CONCLUSION}

In this paper, an identification method is proposed for the identification of linear systems with input switch or backlash nonlinearity. The key step of the algorithm is the design of a specific excitation signal allowing a suitable parametrization of the identification problem. This choice allows the use of subspace identification methods and then the estimation of a model for both the linear part and the non linear part. A numerical example shows the effectiveness of the proposed algorithm. In this paper only the open loop identification problem is considered, we believe that the method developed here can be extended to the closed loop identification problem.

\section{REFERENCES}

[1] E. Bai Identification of linear systems with hard input nonlinearities of known structure. Automatica, 38, 853-860, 2002.

[2] E. Bai and K. Li Convergence of the iterative algorithm for a general Hammerstein system identification. Automatica, 46, 1891-1896, 2010.

[3] L. Bako and G. Mercere and S. Lecoeuche and M. Lovera. Recursive Subspace Identification of Hammerstein Models Based on Least Squares Support Vector Machines. IET Control Theory and Applications, 3, 1209-1216, 2009.

[4] V. Cerone and D. Regruto Bounding the parameters of linear systems with input backlash. IEEE Transactions on Automatic Control, 52, 531536, 2007.

[5] V. Cerone and D. Piga and D. Regruto. Bounded-error identification of linear systems with input and outmut backlash. SYSID, 2012.

[6] F.H.I. Chang and R. Luus A Noniterative Method for Identification Using Hammerstein Model. IEEE Transactions on Automatic Control, 16, 464-468, 1971.

[7] H.F. Chen. Strong Consistency of Recursive Identification for Hammerstein Systems With Discontinuous Piecewise-Linear Memoryless Block. IEEE Transactions on Automatic Control, 50, 1612-1617, 2005.

[8] X.M. Chen and H.F. Chen. Recursive Identification for MIMO Hammerstein Systems. IEEE Transactions on Automatic Control, 56, 895-902, 2011.

[9] A. Chiuso. The role of vector auto regressive modeling in predictor based subspace identification. Automatica, 43, 1034-1048, 2007.

[10] W. Favoreel and B. De Moor and P. Van Overschee. Subspace state space system identification for industrial processes. Journal of Process Control, 10, 149-155, 2000.

[11] F. Giri and E. Bai. "Block Oriented Nonlinear System Identification.". Springer-Verlag, Berlin, 2010.

[12] F. Giri and Y. Rochdi and F.Z. Chaoui and A. Brouri. Identification of Hammerstein systems in presence of hysteresis-backlash and hysteresisrelay nonlinearities. Automatica, 44, 767-775, 2008.

[13] F. Giri and Y. Rochdi and A. Brouri and F.Z. Chaoui. Parameter identification of Hammerstein systems containing backlash operators with arbitrary-shape parametric borders. Automatica, 47, 1827-1833, 2011.

[14] I. Goethals and K. Pelckmans and L. Hoegaerts and J. Suykens and B. De Moor. Subspace intersection identification of Hammerstein-Wiener systems. CDC-ECC, 2005.

[15] J.C. Gómez and E. Baeyens Subspace Identification of Multivariable Hammerstein and Wiener Models. IFAC World Congress, 2002.

[16] Y. Han and R.D. De Callafon Hammerstein system identification using nuclear norm minimization. Automatica, 48, 2189-2193, 2012.

[17] K. Jalaleddini and R.E. Kearney. An identification algorithm for Hammerstein systems using subspace method. ACC, 2011.

[18] T. Katayama. "Subspace methods for system identification". SpringerVerlag, 2005.

[19] T. Knudsen Subspace identification - reducing uncertainty on the stochastic part. IFAC World Congress, Barcelona, 2002.

[20] F. Le and I. Markovsky and C. Freeman and E. Rogers. Recursive Identification of Hammerstein Systems with application to Electrically Stimulated Muscle. Control Engineering Practice, 20, 386 - 396, 2012.

[21] A. Naitali and F. Giri Persistent Excitation by Deterministic Signals in Subspace MISO Hammerstein System Identification. ACC, 2011.
[22] K.S. Narendra and P.G. Gallman An Iterative Method for the Identification of Nonlinear Systems Using a Hammerstein Model. IEEE Transactions on Automatic Control, 11, 546-550, 1966.

[23] S.J. Qin and W. Lin and L. Ljung. A novel subspace identification approach with enforced causal models. Automatica, 41, 2043-2053, 2005.

[24] S.J. Qin. An overview of subspace identification. Computers and chemical engineering, 30, 1502-1513, 2006.

[25] Y. Rochdi and F. Giri and F.B. Gning and F.Z. Chaoui. Identification of block-oriented systems in the presence of nonparametric input nonlinearities of switch and backlash types. Automatica, 46, 864-877, 2010.

[26] P. Van Overschee and B. De Moor. "Subspace identification for linear systems. Theory, implementation applications". Kluver Academic Publishers, Leuven, 1996.

[27] T.H. Van Pelt and D.S. Bernstein. nonlinear system identication using Hammerstein and nonlinear feedback models with piecewise linear static maps. Int. Journal of Control, 74, 1807-1823, 2001.

[28] J.W. Van Wingerden and M. Verhaegen. Subspace identification of Hammerstein-Wiener systems operating in closed loop. Block-oriented Nonlinear System Identification - F. Giri and E. Bai, Springer, 229-240, 2010.

[29] M. Verhaegen. Subspace model identification part 3: analysis of the ordinary output error state space model identification algorithm. International Journal of Control, 58, 555-586, 1993.

[30] M. Verhaegen. Identification of the deterministic part of MIMO state space models given in innovations form from input output data. Automatica, 30, 61-74, 1994.

[31] J. Voros. Modeling and identification of systems with backlash. Automatica, 46, 369-374, 2010.

[32] J. Wang and Q. Zhang and L. Ljung. Revisiting Hammerstein system identification through the Two-Stage Algorithm for bilinear parameter estimation. Automatica, 45, 2627-2633, 2009.

[33] C. Yana and J. Wanga and Q. Zhang. Subspace identification methods for Hammerstein systems: rank constraint and dimension problem. International Journal of Control, 83, 2204-2216, 2010.

[34] W. Zhao and H. Chen. Recursive Identification for Hammerstein System With ARX Subsystem. Transactions Automatic Control, 51, 19661974, 2006.

[35] W. Zhao and H. Chen. Adaptive tracking and recursive identification for Hammerstein systems. Automatica, 45, 2773-2783, 2009. 